\title{
Disonancias entre discurso y realidad empírica de la prostitución
}

\author{
Discordance between discourse and empirical reality \\ regarding prostitution
}

Carmen Delgado

Recibido: 23/01/2018

Aceptado: 29/03/2018

\section{RESUMEN}

Partiendo de la evidencia empírica disponible se cuestiona la validez teórica del modelo regulacionista de la prostitución. La disonancia entre la investigación con muestras amplias y pruebas estandarizadas, y las tesis regulacionistas sustentadas en excepciones, plantea al modelo una contradicción que trata de resolver con estrategias de legitimación dentro y fuera del ámbito académico. El imaginario social lego ha incorporado, sin embargo, sus categorías discursivas tomadas de la lógica neoliberal del libre mercado, como se muestra en el estudio realizado con estudiantes y población general. El discurso articulado en torno a una concepción idealizada, individualista, y despolitizada de la libertad, ha impregnado con éxito el imaginario de la población universitaria. En la estructura de la representación de la prostitución, emerge una dimensión de legitimación "blanda o benevolente", no presente en la población general. Se plantea la necesidad de mayor atención a los procesos formativos de imaginarios sociales legos desconectados de la realidad empírica, y la necesidad de apropiarse de «campos entrecruzados» de conocimiento para abordar el entramado del fenómeno prostitucional.

Palabras clave: prostitución, imaginario social, regulacionismo, abolicionismo

\section{ABSTRACT}

Based on the available empirical evidence, the theoretical validity of the regulationist model of prostitution is questioned. The dissonance between research with broad samples and standardized tests, and the regulatory thesis supported by exceptions, presents the model with a contradiction that it tries to solve with strategies of legitimation, inside and outside the academic sphere. The lay social imaginary has, however, incorporated its discursive categories taken from the neoliberal logic of the free market, as shown in the study carried out with students and the general population. The discourse articulated around an idealized, individualistic, and depoliticized conception of freedom has successfully impregnated the imaginary of the university population. In the

Carmen Delgado es catedrática de Psicometría de la Universidad Pontificia de Salamanca (España). Exdirectora del postgrado "Intervención multidisciplinar en violencia de género" en la Facultad de Psicología y del postgrado "Género y comunicación" en la Facultad de Comunicación. Correo electrónico: cdelgado@upsa.es. ID: https:/ / orcid.org/0000-0002-6757-6317

Cómo citar este artículo: Delgado, C. (2018). Disonancias entre discurso y realidad empírica de la prostitución. Atlánticas. Revista Internacional de Estudios Feministas, 3 (1), 85-112 doi: http:/ / dx.doi.org/10.17979/arief.2018.3.1.3268 
structure of the representation of prostitution, a "soft or benevolent" legitimation dimension emerges, not present in the general population. The need arises for greater attention to the training processes of lay social imaginaries disconnected from empirical reality, and the need to appropriate "cross-linked fields" of knowledge to address the framework of the prostitution phenomenon.

Keywords: prostitution, discourse, social imaginary, regulationism, abolitionism 


\section{ALGUNAS PREMISAS IRRENUNCIABLES PARA UN MARCO TEÓRICO}

Una premisa básica para las ciencias que se aproximan a los fenómenos sociales, es que las teorías que pretenden explicar la realidad deben estar soportadas por evidencias empíricas. No es la capacidad de penetración social lo que da validez científica a una teoría, sino su adecuación a la realidad. En el caso de la prostitución, si este principio se aplicara con rigor no tendríamos dos modelos alternativos debatiendo por mostrar su mayor capacidad explicativa, sino una teoría sustentada por la evidencia empírica y otra que se apoya en la excepción a esta evidencia. Los datos sobre las condiciones materiales de la prostitución son tan contundentemente devastadores, que resulta científicamente inexplicable la pervivencia de la teoría regulacionista ${ }^{1}$, tan ciega y contraria a lo que estos datos muestran. Y, sin embargo, mantener la discusión anclada en las prácticas materiales es extremadamente importante, como bien argumenta Melissa Farley (2009, p. 311).

Los análisis que parten de los datos macrosociales muestran la confluencia de neoliberalismo y patriarcado para producir la dominación y explotación de las mujeres en el sistema prostitucional ${ }^{2}$. Rosa Cobo (2017) analiza la coincidencia de intereses patriarcales y neoliberales con el crecimiento exponencial de la mercantilización del cuerpo de mujeres y menores, a través de la pornografía, la prostitución y la trata que suministra mujeres y niñas al sistema. Otros trabajos como los de Ana de Miguel (2015) o Beatriz Gimeno (2012), han analizado también esta coincidencia de intereses que institucionaliza la subordinación de las mujeres y la disponibilidad de sus cuerpos para uso de los hombres. El pensamiento feminista así lo ha entendido históricamente, y reivindicado por ello la abolición de esta institución patriarcal basada en relaciones de poder y en modelos de sexualidad de centralidad masculina (Pedernera y Torrado, 2015). Pero la irrupción del orden neoliberal ha alterado no sólo las condiciones materiales de las mujeres y de las mayorías empobrecidas del planeta, sino que ha penetrado en algunos sectores del pensamiento feminista, permeabilizando sus categorías de análisis. Nancy Fraser (2015) analiza las derivas de los ideales

\footnotetext{
${ }^{1}$ En este trabajo se adoptará el término regulacionista para referirse a toda forma de legalización, independientemente del grado de reglamentación. Para una revisión de diferentes modelos normativos, puede verse, por ejemplo, el trabajo de María José Barahona (2015).

2 Laura Pedernera y Esther Torrado (2015, p.1385) tras revisar distintas acepciones del término prostitución, justifican desde la sociolingüística la pertinencia de considerarla un sistema, en cuanto que sólo tiene sentido dentro del contexto social que la propicia, respalda y favorece.
} 
feministas que, buscando profundizar la justicia de género, acabaron sirviendo a los fines neoliberales. Las coincidencias del actual modelo regulacionista con los valores del libre mercado, es un buen exponente de este resultado. Categorías como transacción comercial, ley de oferta y demanda, libre intercambio de bienes, clientes y consumidores, compra y venta de sexo, etc. han penetrado en el marco analítico del feminismo regulacionista, en una forma muy coincidente con lo que Zygmunt Bauman (2000) tematiza como "modernidad líquida". La incapacidad de resistirse a las reglas del juego y a los criterios de racionalidad inspirados y moldeados por el mercado, característicos de la nueva modernidad, armonizan muy sintónicamente con el regulacionismo. De modo que la aseveración de Fraser (2013) en The Guardian, resuena con toda su potencialidad ante esta construcción legitimadora de la prostitución:

"el movimiento por la liberación de las mujeres se ha enredado en una relación peligrosa con los esfuerzos neoliberales para construir una sociedad de libre mercado y las ideas feministas que formaron parte de una cosmovisión radical de la igualdad, se expresan cada vez más en términos individualistas (neo) liberales".

La recurrencia al mutuo acuerdo entre dos partes igualmente interesadas y libres, que intercambian servicios (trabajadora sexual) por dinero (cliente), sin que importe la posición de los intervinientes ni las condiciones en que se efectúa, ni la equiparabilidad del valor de lo que se intercambia, etc. evoca la imagen de un "feminismo líquido" diluyéndose en la nueva modernidad. Si nada hay que objetar a la ley del libre mercado, si todo es resignificable sin que la resignificación implique cambios en las condiciones materiales, si lo real es la representación mental sin una traducción en lo concreto, entonces todo se reduce a validar discursos en lo abstracto. Pero para quienes pensamos desde disciplinas empíricas, las explicaciones teóricas o bien se construyen sobre datos, o carecen de validez. Cuando la observación de campo hace irreconocible la imagen del fenómeno prostitucional dibujada por el regulacionismo -y así se expondrá más adelante-, no hay forma de entender su explicación de la prostitución, y una explicación que no se entiende no es una explicación, dice Sandra Harding (1986).

Para el regulacionismo todo queda legitimado si ambos intervinientes 
participan "formalmente" en la transacción prostitucional; una premisa a la que nada pueden objetar los intereses neoliberales, como de hecho sucede. Empresarios del negocio prostitucional no sólo se suman a este postulado, sino que lo reconocen y abanderan como suyo. Pero el regulacionismo que aspira a definirse como feminista necesita introducir la categoría del género en el discurso, y lo hace tematizando la desmitificación de la sexualidad, y la transgresión como emancipación que se actualizaría en la prostitución. Así, la disponibilidad sexual para los hombres se resignifica como "agencia de las mujeres", la fijación de un precio para este acceso se resignifica como apropiación de un "nicho laboral" que empodera, y la interseccionalidad de etnia y clase social con género se resignifica como "posibilidad de migración" para mujeres pobres de países empobrecidos. El problema para el regulacionismo no es la prostitución; el problema es la estigmatización de las mujeres que optan por esta profesión, a la que contribuiría el feminismo abolicionista.

\section{DE LO DISCURSIVO A LO EMPÍRICO Y VICEVERSA}

Organizaciones como COYOTE, acrónimo de «Call Off Your Old Tired Ethics». contribuyeron a introducir la idea de que la posición progresista ante la prostitución es defender su pervivencia insertándola en el marco de los derechos civiles, la libre elección y la regulación del trabajo (Jenness, 1990). Esta visión idílicamente transgresora introdujo una representación del fenómeno prostitucional, que conecta con un marco de emancipación sexual libre ipor fin! de su asociación histórica con el pecado, el crimen y el sexo ilícito. Pero además de rescatarla del estigma social y de otorgarle el estatuto de opción profesional, el regulacionismo se erige en conquista histórica del nuevo feminismo, puesto que la legalización aportaría la legitimidad para que las mujeres puedan usar sus cuerpos como quieran, con quien quieran y para lo que quieran. La prostitución les devolvería así ipor fin! la agencia usurpada por el feminismo abolicionista y antipornografía, al que se supone instalado en lo que Judith Butler (1997, p. 36) denomina un "marxismo neoconservador". En suma, el discurso proprostitución aspira a implantar en el imaginario social la idea de que el problema es de orden cultural, pues todo se reduce al estigma social. El nuevo feminismo postmoderno y queer será el que habrá de liberar la prostitución de discursos desfasados y anclados en los rancios postulados de una sexualidad sometida y patriarcal. En obras como "Putas y Otras 
Feministas" (Nagel, 1997), se exponen los beneficios de la empoderante resignificación de la prostitución, y se loan los beneficios del nuevo "trabajo sexual" que mejorará la vida de muchas mujeres (Leigh, 1997).

Esta representación ha penetrado con suma facilidad en el imaginario social $\operatorname{lego}^{3}$ y ha incidido con mayor éxito en la población universitaria que, sin mayores reflexiones sobre el tema, asocia prostitución con ideas progresistas.

En una investigación en curso, una muestra incidental de 2.266 personas entre 15 y 87 años (62,5\% mujeres y 37,5\% hombres) respondió a un cuestionario sobre diversos aspectos sociales, incluyendo opiniones sobre la prostitución. Mediante la prueba chi cuadrado encontramos una diferencia estadísticamente significativa $(\mathrm{p}=.000)$ en la posición declarada por estudiantes y población general:

Tabla 1.- Posición ante la prostitución en estudiantes y en población general

\begin{tabular}{|l|l|l|l|}
\hline Prostitución & $\begin{array}{l}\text { A Favor } \\
\text { Muy a Favor }\end{array}$ & Indiferente & $\begin{array}{l}\text { En Contra } \\
\text { Muy en Contra }\end{array}$ \\
\hline Estudiantes $(\mathrm{n}=573)$ & $45,2 \%$ & $32,7 \%$ & $22,1 \%$ \\
\hline Población General $(\mathrm{n}=1693)$ & $24,8 \%$ & $42,6 \%$ & $32,6 \%$ \\
\hline
\end{tabular}

Teniendo en cuenta que se trata de muestras que no han hecho ninguna lectura específica sobre el tema, podemos tomar estos datos como representativos de un "imaginario lego". En el "imaginario profesional" obtenido por María José Barahona (2015) con una muestra de 450 profesionales de Trabajo Social, la posición declarada es mayoritariamente contraria, principalmente prohibicionista y muy poco abolicionista (p.115). Esta posición declarada no coincide, sin embargo, con la actitud evaluada a través de escalas. En este caso, el abolicionismo obtiene porcentajes de aceptación abrumadoramente superiores a cualquier otra posición (p. 119-123). La proximidad profesional a la realidad de la prostitución ¿haría inclinar la balanza hacia el abolicionismo, cuando no hay una teoría previa que validar? Lo que sí parece obvio es que la "gente común" la percibe con categorías más simples como "a favor" o "en contra", sin tener demasiado clara la diferencia entre abolir o prohibir, regular o

\footnotetext{
3 El término "lego" se utiliza aquí en el sentido teorizado en la tradición psicológica (Kelly, 1955; Heider, 1958), para referirse a teorías de sentido común o teorías ingenuas, con las que las personas elaboran sus explicaciones de la realidad. Esta perspectiva abrió una importante línea de investigación en diferentes campos de la Psicología, como las Teorías Implícitas de la Personalidad. Para una interesante revisión sobre su impacto en los procesos de percepción social y relaciones intergrupales, puede consultarse el trabajo de Laura Ramírez y Shery Levy (2010), disponible en http://revistas.javeriana.edu.co/index.php/revPsycho/article/view/744
} 
legalizar.

En nuestra muestra, con las características que se acaban de indicar, al cruzar el posicionamiento ante la prostitución con la ideología mediante un Análisis de Correspondencias ${ }^{4}$, se observa una relación estadísticamente significativa $(p=.001)$ entre estas dos variables. En el gráfico siguiente se muestra la proximidad entre ideología progresista y posición favorable o muy favorable, así como la proximidad de la ideología conservadora con posición muy contraria. A medio camino, para la ideología moderada la prostitución es algo indiferente o se posiciona en contra:

Figura 1. Relación entre ideología y posición ante la prostitución

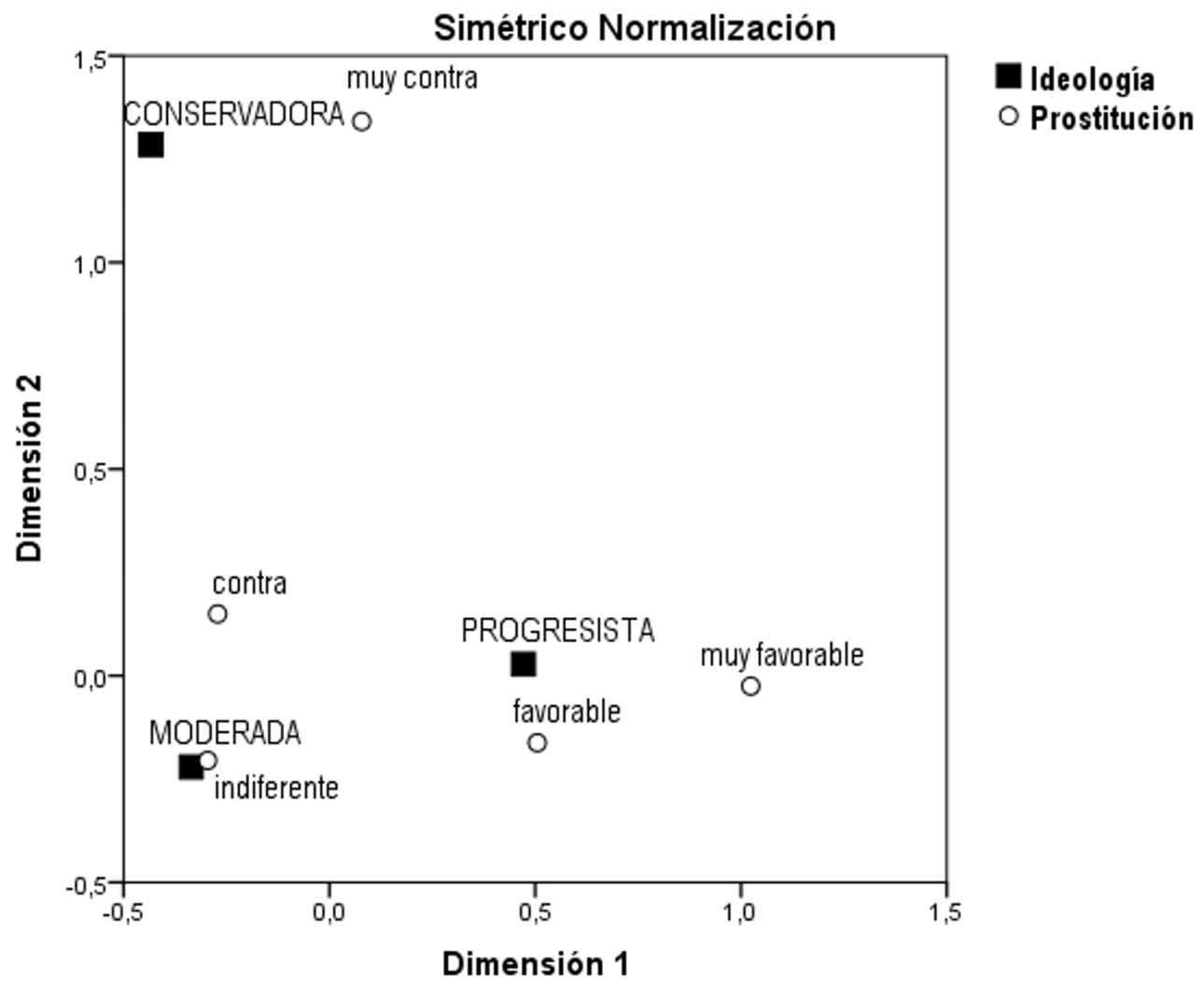

No cabe duda de que, a nivel discursivo, el regulacionismo ha sabido visibilizar las ventajas que se seguirían de la legalización de la prostitución, con mayor éxito que el abolicionismo mostrar su nocividad para las vidas de las mujeres. Ha sabido construir una narración persuasiva revestida de modernidad,

\footnotetext{
${ }^{4}$ En este trabajo se omiten las cuestiones técnicas de los análisis estadísticos, tomando únicamente lo relevante para su interpretación. Para una profundización de las técnicas puede verse, por ejemplo, Delgado (2014).
} 
aunque la realidad empírica en nada se parezca a la liberadora imagen de una "profesión como otra cualquiera», de la que el $89 \%$ de las mujeres está buscando activamente salir de ella sin lograrlo por falta de alternativas reales y por el fracaso de la restitución vincular tras la experiencia traumática del ejercicio (Baker, Dalla, y Williansom, 2010). Más que una profesión "libremente elegida" se revela como un "modo de sobrevivencia impuesto" por la trata o por la necesidad.

La huella psicológica de la prostitución muestra niveles de vulnerabilidad, estado de alerta, pérdida de control, ansiedad, deterioro físico, trastornos de alimentación, pérdida de autoestima, disociación, estrés postraumático, aislamiento, estados depresivos, trastornos del sueño, alteraciones emocionales, ideación suicida e intentos de suicidio... en absoluto comparables a los encontrados en cualquier otro trabajo (Farley, 2017; Hedin y Mänsson, 2003; Mänsson y Hedin, 1999). Melissa Farley (2003, p.5) enumera más de 30 publicaciones que documentan cómo la violencia sexual y la agresión física constituyen las experiencias comunes de las mujeres en la prostitución. Trabajos de campo con muestras amplias sacan a la luz el abuso sufrido, incluyendo la violación en el 70\% de ellas (Silbert y Pines, 1982).

Esta situación, lejos de mejorar en los países que han optado por la legalización, aparece tozudamente desalentadora desmintiendo que el "trabajo sexual" sea como los demás. El informe publicado por el propio Ministerio de Seguridad y Justicia de Holanda (Ministerie van Justitie in Veiligheid, 2015) a partir del trabajo de campo realizado en 2006, afirma que las condiciones de vida de las mujeres en prostitución son menos saludables que las del resto de holandeses. Lo que sí gana en salud, por el contrario, es la economía del país que con la prostitución legal ingresa 2.500 millones de euros, lo que supone el 0,4\% del PIB. Más salud para la economía, menos para las mujeres. Y la misma situación se reporta en Alemania, con estudios como el realizado en 2004 por Schröttle (Kraus, 2016) en el que el 92\% de las mujeres prostituidas sufre acoso sexual, casi el 90\% violencia física y psicológica, y el 59\% violencia sexual.

\section{ESTRATEGIAS (PSEUDO)REFUTACIONISTAS DE LA REALIDAD EMPÍRICA}

El papel preponderante de la libertad individual en el discurso regulacionista, 
favorece sin duda la mayor visibilización de lo personal al situar en una posición central el bienestar de las personas, sea o no real. Es posible, y habría que someterlo a contrastación empírica, que el imaginario social lego se nutra más de las ideas sobre la incidencia concreta en la vida concreta de las personas concretas que, sobre valores abstractos como la justicia, equidad, o dignidad de las personas. En los últimos años hemos podido observar cómo la movilización política entre la población joven responde más a causas concretas que a reivindicaciones abstractas. En el tema de la prostitución disponemos de teorías estructurales que ofrecen sólidos análisis a nivel macro; pero quizás tenga razón Lara Gerassi (2015) al reclamar mayores aportes en el nivel micro -aunque presta poca atención a los ya existentes-, que visibilicen los efectos de la prostitución en la vida de las personas y en sus relaciones. Esta preponderancia de los análisis estructurales ha tenido incidencia en la discusión política de los países que deben optar por un marco normativo en su ordenamiento jurídico; pero ha penetrado menos en el imaginario social de una población que no tiene mayor interés en profundizar en un problema que considera ajeno a su vida. Quizás por eso, iniciativas como la de Teresa Lozano y Zua Méndez (TowandaRebels, 2017) han encontrado amplio eco y provocado numerosas reacciones. El video "Hola, putero" que entró en diciembre en las redes sociales, además de conseguir su objetivo de visibilizar al protagonista oculto de la prostitución, tiene el mérito de traducir con éxito la realidad de la vida de las mujeres prostituidas. Coincidimos pues con Gerassi (2015) en señalar la necesidad de más análisis micro sobre los procesos de entrada, mantenimiento y salida de las mujeres y niñas en el sistema prostitucional; pero necesitamos también investigar de qué modo los datos ya disponibles pueden entrar en los imaginarios sociales e incidir en la opinión pública, además de sacar del ángulo ciego de este imaginario, al demandante de la prostitución y sus narrativas de sexo líquido, como analiza Rosa Cobo (2017).

El panorama regulacionista, como se acaba de señalar, en nada se parece a la realidad empírica de la prostitución, a tenor de los datos disponibles. La huella psicológica está tan presente en la legal como en la clandestina, y en la de calle como en la de interior; la prostitución resulta traumatizante bajo el paraguas de la legalización, y fuera de él. Las mujeres aprecian poco los prometidos beneficios de la legalización, puesto que la mayoría elige no "regularse" en los países en que pueden hacerlo. 
No hay forma de sostener, por tanto, la imagen empoderante y liberadora de la legalización. Lo que se anunciaba como la posibilidad de agencia de las mujeres ${ }^{5}$, resultó ser la posibilidad de mayor explotación y beneficios para la industria prostitucional como muestra la evidencia de que "la prostitución legal está asociada con el aumento de tráfico. Traficantes y proxenetas pueden operar con mayor impunidad cuando la prostitución es legal (...) A día de hoy el 80\% de todas las mujeres en prostitución en Alemania y Holanda son traficadas" (Farley, 2009, p. 313). Quienes presentan la prostitución como un objeto más de consumo, así lo señala hábilmente Melissa Farley (2017), tendrían que saber que las teorías sobre consumo no se cumplen cuando la persona es el producto; por tanto, la prostitución no cabe en este marco explicativo.

Ante la refutación empírica del discurso regulacionista, sólo parecen abrirse entonces dos posibilidades: la reformulación, o la introducción de algún factor de corrección para mantener inalterados sus postulados. Y, como era de esperar, se opta por lo segundo. En el ámbito del activismo extra-académico se adopta una táctica negacionista, apelando a testimonios de mujeres que declaran su opción libre y su vida saludable en el sistema prostitucional, así como su deseo de mantenerse en lo que consideran su profesión (véase por ejemplo Mertuil, 2017). Se utiliza el "caso único" (testimonios individuales) para refutar el "estudio poblacional" (muestras de diferentes formas y tamaños). No es un modo metodológicamente ortodoxo de proceder; pero el impacto en el imaginario social no depende de la corrección del método, sino de la visibilidad y saliencia que consiga imprimir en la población. Y, desde luego, no se puede negar la potencialidad de sus acciones para impactar el imaginario social. Lo excepcional, extravagante o rupturista, siempre capta la atención de los medios, y sus acciones ciertamente tienen estas características ${ }^{6}$. Pero además de los aspectos formales, hay una cuestión de fondo que explica el éxito: el

\footnotetext{
${ }^{5}$ El tema de la agencia de las mujeres en la prostitución es revisado por Sheila Jeffreys (2012), analizando las contradicciones entre la definición adoptada por el regulacionismo como "libertad de acción de la mujer prostituida", y las relaciones opresivas de poder dentro de las cuales actúa. Jeffreys analiza el modo en que esta definición de "agencia" se inserta en el requerimiento neoliberal de relatos en los que las personas narren su historia de vida como si fuera el resultado de elecciones deliberadas. El subtexto que subyace en esta definición, continúa Jeffreys, es la incuestionabilidad del "derecho sexual masculino", al cual sirve la prostitución como "una práctica de género por excelencia" (p.65). El feminismo comenzó a usar el término "agencia" para enfatizar que las mujeres no eran sólo víctimas pasivas de las fuerzas históricas, y que podían oponer resistencia a las estructuras de poder, no sólo individualmente, sino colectivamente en los movimientos sociales. Lamentablemente el concepto resultó ser un "regalo envenenado", concluye Jeffreys, pues para algunos sectores del feminismo se redujo al intento de sobrevivir y encontrar cualquier forma de evitar la pobreza o mantener la vida; pero la supervivencia no es lo mismo que la resistencia o el intento de transformar las relaciones de poder (p.59).

${ }^{6}$ Véase por ejemplo "Cursos de prostitución profesional con trabajo asegurado al final de la formación" puesta en marcha en 2012: http://www.que.es/ultimas-noticias/curiosas/201205081825-cursosprostitucion-profesional-trabajo-asegurado-cont.html
} 
imaginario patriarcal de la sociedad no ofrece ninguna resistencia para incorporarlo, puesto que resulta muy sintónico con no tocar la prostitución, salvo que sea para potenciarla. Así pues, todo es favorable para un anclaje fácil y rápido.

En el ámbito académico se requiere otra estrategia para resolver la disonancia entre discurso y realidad empírica, puesto que las publicaciones científicas exigen "un mínimo" de corrección metodológica. La vía elegida es precisamente la crítica de la metodología utilizada en los estudios empíricos y ¡sorprendentemente! esta crítica se construye desde la más genuina tradición positivista, apelando a la no-neutralidad de las investigaciones. Así, por ejemplo, la sucesión de artículos de Ronald Weitzer ilustran cómo el único núcleo argumentativo se reduce al cuestionamiento de los resultados, apelando ¡al activismo social de las investigadoras! Claro que habrá de sobreentenderse "activismo abolicionista", teniendo en cuenta que quien hace tal crítica es un laborioso "activista regulacionista". En el artículo titulado "Teoría y Método Defectuosos en Estudios de Prostitución" (Weitzer, 2005) se cuestionan los resultados obtenidos por Jody Raphael y Deborah Shapiro (2004), Melissa Farley (2004), y Janice Raymond (2004). El punto de partida de la crítica es que los tres artículos están escritos por "autoras que adoptan la versión extrema de la teoría feminista radical, extrema en el sentido de que es absolutista, doctrinaria y no científica" (p. 934); es decir, una teoría "defectuosa" que utiliza un lenguaje inadecuadamente "tendencioso" pues, por ejemplo, denomina a los clientes "usuarios de prostitutas o depredadores sexuales", y a las trabajadoras sexuales "mujeres prostituidas o sobrevivientes". Esta resistencia a usar el término "prostitutas", denominación neutra en opinión de Weitzer, denota la estigmatización de las trabajadoras sexuales por parte del feminismo radical. El defecto teórico, por tanto, se resume en una enmienda a la totalidad del modelo abolicionista, sin más. Pero si la teoría es defectuosa, la metodología también lo es, y la razón es que estas autoras seleccionan "los peores ejemplos disponibles" y presentan "las anécdotas" como evidencia que es interpretada como coacción y desigualdad. Son las investigadoras (no el ejercicio de la prostitución) las que victimizan a las trabajadoras sexuales bajo la etiqueta de explotadas y, en suma, los estudios realizados desde el abolicionismo violan los criterios de un pensamiento científico (p. 937). Hemos de sobrentender en buena lógica, que también los informes recientes que dibujan idéntico panorama empírico, como el publicado por el Parlamento Europeo (Schulze, Novo, Mäson, y Saklin, 2014), 
adolecen de los mismos defectos teóricos y metodológicos. En este informe se estima que lo que Weitzer califica como "anécdotas" de violencia física y de "los peores ejemplos disponibles", está presente en el $92 \%$ de las mujeres prostituidas, habiendo intentado el suicidio el 25\% de ellas, sin mencionar la violencia emocional mucho más devastadora según señalan los expertos y expertas (véase por ejemplo Klaus, 2016).

Pero examinemos los métodos supuestamente defectuosos de los trabajos empíricos, los únicos que nos permiten acceder a la realidad "contante y sonante" de la prostitución. Nada de lo que se señala como "defecto" tiene entidad para amenazar la validez de los resultados; pero además ninguna de las objeciones es aplicable al conjunto de las investigaciones, coincidentes en confirmar el daño psicosocial de la prostitución. Los "defectos" señalados no son aplicables al conjunto de estudios, sino que se van elaborando específicamente para cada uno; esto es, son objeciones ad hoc para cada investigación 7 . Lo cierto es que los estudios descalificados por Weitzer utilizan instrumentos estandarizados y muestras amplias, utilizan muestreos no aleatorios característicos de este tipo de estudios, indican el procedimiento de obtención de datos, e incluyen en las muestras diferentes estratos de edad, raza, tipo de prostitución; en suma, siguen la metodología habitual en este tipo de estudios (Farley, 2005). ¿Dónde podríamos encontrar entonces lo "defectuoso" del método, que invalidaría los resultados de las investigaciones empíricas tan poco convenientes para las aspiraciones regulacionistas? Weitzer (2005, p. 937938) lo condensa en estos tres grandes argumentos: (1) algunos estudios no reportan cómo y dónde se contactan los sujetos entrevistados, (2) otros no incluyen grupos de comparación, y (3) en otros, las entrevistadoras son "exprostitutas". En cuanto al (1), no reportar dónde ni cómo, no sólo no es un defecto metodológico, sino que es un resguardo ético obligado cuando implica riesgo para las personas participantes. En cuanto a (2) ausencia de grupo de comparación, entre la variedad de diseños de las investigaciones ex-post-facto propios de las ciencias sociales, el cuasi-experimental sin grupo control al que se refiere Weitzer, está legitimado metodológicamente para proporcionar descripciones como las que ofrecen estos estudios (León y Montero, 2010).Pero además, ¿no es suficiente "grupo de comparación" la población general, en la que las tasas de sintomatología es ostentosamente menor?. Y respecto a (3)

\footnotetext{
${ }^{7}$ Esta estrategia de crítica no sistemática, específica para cada caso, pudiendo contradecirse incluso de un caso a otro, es una de las estrategias post-machistas analizadas por Miguel Lorente: http://blogs.elpais.com/autopsia/2013/05/el-posmachismo-i.html
} 
inadecuación de las entrevistadoras por tratarse de exprostitutas, lo que el autor considera un inconveniente resulta ser una ventaja epistemológica en las corrientes que hace tiempo han reemplazado al positivismo, en el que parece permanecer instalado Weitzer (véase por ejemplo Haraway, 1988). La condición de haber ejercido la prostitución no sólo no es un inconveniente, sino que otorga una posición epistémicamente privilegiada, tanto para facilitar una comunicación significativa con y entre las participantes, como para comprender las experiencias relatadas. Pero lo llamativo de esta objeción, es que provenga de quien afirma que la prostitución es un trabajo como otro cualquiera ¿Hemos de sospechar, acaso, que hay algo "incapacitante" en ella, que no se nos ha contado?

La cuestión de la "neutralidad" como argumento, resulta poco rigurosa. La imposibilidad de observar la realidad sin "algún punto de vista" ha sido ampliamente fundamentada, tanto desde filosofías feministas (por ejemplo, Harding, 2004; 2009) como no feministas (por ejemplo, Martín Baró, 1990) ${ }^{8}$. Igual de "no-neutra" es la mirada de quienes han ejercido la prostitución, que la de quienes la consideran un trabajo sexual como otro cualquiera; y de ningún otro modo podría ser. Como han mostrado los estudios clásicos de la psicología cognitiva, la percepción humana es selectiva y está mediada por categorías interpretativas; no hay otra forma de percibir, psicológicamente posible. No hay posibilidad humana de "percepción neutra". La percepción que somos capaces de procesar cognitivamente, nos viene en forma de paquete compacto: estímulo y significado del estímulo9. Podemos afirmar, por tanto, que argumentar falta de neutralidad en las investigaciones que se desarrollan en proyectos que adoptan un marco interpretativo que considera la prostitución como una forma de violencia contra las mujeres y no una industria legítima (Weitzer, 2005, p. 939), es cuanto menos, poco serio, por no decir poco ético ¿Habrá de entenderse acaso, que lo "no erróneo" es investigar bajo marcos interpretativos que consideran la prostitución una industria legítima? ¿O será acaso lo correcto, que los proyectos no expliciten su marco interpretativo y mantengan la opacidad, bien conocida, de los valores con los que se aproximan a la realidad? Todo proyecto científico, como, señala Melissa Farley (2005) en su réplica, está

\footnotetext{
${ }^{8}$ Para una aproximación a la objetividad científica véase, por ejemplo, el trabajo de Zenaida Yanes (2012) en el que analiza este concepto través de cuatro autoras feministas: Sandra Harding, Evelyn Fox Keller, Donna Haraway y Helen Longino.

${ }^{9}$ La obra traducida al castellano del psicólogo Daniel Kahneman (2014) es una excelente monografía para comprender cómo funciona la psicología cognitiva (aunque no hay en ella ningún atisbo de conciencia de género).
} 
impregnado de valores; desde la investigación de células madre, hasta la investigación de los efectos psicológicos de la colonización de un pueblo por otro, o de los efectos del incesto, la violación o la prostitución. El problema no es si la investigación está impregnada de valores, siempre lo está; el problema es si esos valores se han explicitado, o se silencian bajo la pantalla de la "neutralidad". Cuando un punto de vista pretende no tener lugar alguno, es porque está situado en el lugar hegemónico que no requiere ser explicitado puesto que se le supone "natural". El interés legalizador de Ronald Weitzer, y de acabar con la prostitución "de calle" (la nociva) en favor de la prostitución "de interior" (la inocua), nada tiene de neutro. Su "neutral" interés por la seguridad que aportaría esta medida, nada tiene de neutral. La seguridad que importa, como él mismo ha expuesto en alguno de sus trabajos (Weitzer, 1994), no es la de las mujeres prostituidas, sino la de las comunidades afectadas por los inconvenientes de convivir con la prostitución. Como siempre, tras el viejo reclamo de la "neutralidad" emerge la alianza ideológica con la posición dominante, y será por esto quizás que, en el caso de la prostitución "los investigadores parecen identificarse más fácilmente con los clientes que con las prostitutas" (Vanwesenbeeck, 1994, p. 33).

Es evidente que la investigación empírica contradice tan radicalmente como el feminismo radical, una imagen benevolente de la prostitución construida como trabajo sexual. No hay "teoría y método defectuosos" en los trabajos que aportan datos contundentes de los efectos devastadores de la prostitución, objetivados, por ejemplo, en el estrés postraumático complejo que sufre el 68\% de mujeres prostituidas (Kraus, 2016). Las investigaciones lideradas por Farley durante décadas, recopilan centenares de entrevistas en países tan diversos como Estados Unidos, Canadá, Colombia, Alemania, México, Sudáfrica, Tailandia, Turquía o Zambia, y muestran que no es anecdótico el daño psicológico. Los datos desmienten que sea la calle -y no la prostitución- lo que ocasiona este daño, desmienten que éste sea cualitativamente diferente al causado por el tráfico o la trata, desmienten que la mayoría de las mujeres elija prostituirse, y desmienten que la legalización y la descriminalización disminuya este daño (Farley et al., 2003). La pregunta, entonces, es otra: ¿por qué esta cuestión no tiene la visibilidad en el imaginario social de una población que, seguramente, pondría el grito en el cielo si los datos provinieran de otro sector poblacional? 


\section{APROXIMACIÓN A UN IMAGINARIO LEGO DE LA PROSTITUCIÓN}

Personas a favor y en contra de la prostitución difieren lógicamente en los argumentos que esgrimen para explicar o justificar su posición. Pero bajo el repertorio de los distintos argumentarios, lo realmente interesante es identificar cuáles de sus elementos discriminan entre una y otra posición. Se trata, pues, de explorar el núcleo discursivo que verdaderamente marca las diferencias, porque es en ese núcleo donde podremos encontrar pistas sobre lo realmente significativo para el posicionamiento de una población. A nivel empírico, podemos acceder a este núcleo a través de diferentes técnicas, siendo una de ellas la exploración de las relaciones que se establecen entre los distintos aspectos de la prostitución. A partir de estas relaciones podemos rastrear su potencial discriminante.

Sabemos que las creencias se aglutinan en dimensiones de mayor nivel de abstracción, que dan forma a una representación mental. En estas dimensiones, no todos los elementos cobran la misma relevancia en la imagen resultante. El peso que adquieren en la representación mental refleja, en último término, la construcción ideológica del fenómeno. La divergencia entre las posiciones abolicionista y regulacionista muestra el diferente peso atribuido a los distintos elementos del fenómeno prostitucional. Mientras para una la violencia y explotación sexual adquiere la mayor relevancia y transversaliza las demás variables, para la otra es la libertad del mercado lo que subyace en la representación de la prostitución. Johanna Niemi (2010) mostró cómo en la representación accesible a través del análisis del discurso, emerge el nivel simbólico en el que operan los valores patriarcales, y cómo modificar el nivel simbólico es condición necesaria para que las actuaciones en el nivel político o social encuentren resonancia en una población. Lo mismo puede decirse en sentido inverso: la modificación del simbólico social impulsa o frena las actuaciones políticas y sociales. El caso de Finlandia analizado por Niemi, nuestra cómo el diferente tratamiento penal de la prostitución, en función de que hubiera o no hubiera tráfico, resultó ineficiente para el propósito de la ley (Niemi y Aaltonen, 2017). Esta distinción mantenía intacta la legitimación de la prostitución en el nivel simbólico, puesto que lo único ilegal era el proxenetismo o tráfico. La modificación del simbólico es tan necesaria como la modificación de los comportamientos para producir cambios, como señala Rita Segato (2010) a propósito de la antropología del género: 
"es necesario minar, desgastar, desestabilizar los cimientos y la ideología que de ellos emana, para producir cambio" (p.70).

Así pues, para acceder empíricamente a la vigencia del simbólico patriarcal en una sociedad resulta útil explorar sus representaciones mentales. Al analizar el peso que adquieren los distintos argumentos, a favor y en contra de la prostitución, podemos observar dónde se ubica el centro sobre el que gravita el posicionamiento en un momento dado. La técnica estadística de Análisis Discriminante, entre otras, nos permite identificarlo.

En una investigación realizada con 239 estudiantes de Psicología (Delgado y Gutiérrez, 2014) se les pidió que valoraron de 1 a 5 la fuerza argumentativa de 22 razones para posicionarse ante la prostitución, independientemente de cuál fuera su posición. Al final del cuestionario se les preguntaba la posición: 50\% se declararon a favor, 34\% indiferentes y sólo un 16\% en contra. Los 22 enunciados que debían evaluar habían sido extraídos de 9 grupos de discusión realizados con 71 estudiantes, 36 mujeres y 35 hombres (Delgado y Gutiérrez, 2015; Gutiérrez, 2017). El Análisis Discriminante mostró que, si bien las valoraciones diferían dependiendo del posicionamiento en el sentido esperado, sólo 4 razones discriminaban significativamente $(p=.000)$ entre quienes se declaraban "a favor" o "en contra". En la tabla siguiente ${ }^{10}$ se muestra este resultado en el que se observa, que 3 de los argumentos discriminantes son "a favor" y 1 en contra:

Tabla 2.- Argumentos discriminantes entre posición "a favor" (F) y “en contra" (C)

\begin{tabular}{|c|c|c|}
\hline \multirow[b]{2}{*}{ Coeficientes de las funciones canónicas discriminantes } & \multicolumn{2}{|c|}{ Función } \\
\hline & 1 & 2 \\
\hline $\begin{array}{l}\text { F7_La prostitución cumple una función social, resuelve el problema de algunas } \\
\text { personas que no podrian tener sexo de otra forma }\end{array}$ & .293 &,- 62 \\
\hline $\begin{array}{l}\text { F3_Mantener y regular la prostitución aportaría beneficios económicos al país; ya } \\
\text { que se recaudarian impuestos por un trabajo que ahora no contribuye fiscalmente }\end{array}$ & 254 & 90 \\
\hline $\begin{array}{l}\text { F9_La prostitución no es denigrante para las mujeres que la ejercen; si una mujer } \\
\text { así lo ha decidido libremente nadie tiene derecho a juzgarla }\end{array}$ &, 423 &,- 14 \\
\hline $\begin{array}{l}\text { C4_Si se legalizara la prostitución se le estaría legitimando éticamente, porque la } \\
\text { gente ve bien lo que es legal; lo que habria que hacer es acabar con la } \\
\text { prostitución, no que la gente la vea bien }\end{array}$ &,- 367 &, 26 \\
\hline (Constante) & $-1,8$ & $-1,5$ \\
\hline
\end{tabular}

Coeficientes no tipificados

10 El coeficiente de la función indica el peso discriminante del argumento, de modo que a mayor coeficiente mayor relevancia. El signo negativo del argumento en contra, indica que la relación con los argumentos a favor es inversa, como es lógico. La función 1 tiene un poder explicativo del 87,4\%, mientras que la función 2 explica el 12,6\%; por tanto, es la función 1 la de mayor interés interpretativo. Para una mayor comprensión de las técnicas estadísticas, puede verse por ejemplo Delgado (2014). 
¿Cómo interpretar estos resultados? En primer lugar, se puede observar que el único argumento "en contra" (C4), alude a la implicación simbólica de legalizar la prostitución: legalizar es legitimar, y el objetivo no es legitimar sino erradicar. Encontramos por tanto una pista muy relevante para aproximarnos a los diferentes imaginarios de una y otra posición; entre los argumentos contrarios, es éste el que marca la diferencia. Otras razones "en contra", resultaron periféricas para situarse ante la prostitución: nunca es realmente libre, tiene consecuencias negativas para la salud de las mujeres, no es liberación sexual sino pseudomodernidad, no es un trabajo más, es explotación económica de la que otros se lucran, estigmatiza a las mujeres, deshumaniza a las mujeres...

En el caso de las razones "a favor" resultaron discriminantes tres (F7, F3, F9), lo que nos indica ya de entrada, que el núcleo discursivo pro-prostitución se simboliza con mayor riqueza de contenidos; incorpora mayor diversidad de razones ofreciendo mayores puntos de anclaje para su aceptación. Esta mayor diversificación abre, por tanto, más puntos de entrada para la adherencia a sus enunciados. Al examinar el contenido, encontramos que se refieren a: (1) no es denigrante para las mujeres, (2) cumple la función social de proporcionar sexo a quienes no lo obtendrían si no existiera, y (3) mejoraría la economía porque generaría ingresos fiscales si se legalizara. Las otras razones "a favor" enunciadas en los grupos de discusión y que fueron evaluadas por la muestra, resultaron periféricas: es un acuerdo libre entre personas adultas, una forma de ganarse la vida como otra cualquiera, es una cuestión de moral o ética individual, se aceptaría si el sexo no fuera tabú, es una transacción comercial como otras, regularla garantizaría los derechos sociales de quienes la ejercen, es inevitable pues satisface necesidades de la especie humana... Así pues, el núcleo de la representación favorable a la prostitución emerge articulado en una clara instrumentalización de las mujeres para proporcionar el sexo que no podría tenerse de otra manera (se sobreentiende, los hombres), para obtener ingresos fiscales beneficiosos para la economía del país, y para rechazar su carácter denigrante.

A la luz de estos resultados, podemos concluir que los datos empíricos contraponen un núcleo argumentativo muy definido: oponerse a la legalización para no legitimar en la posición contraria, frente a nada hay de malo en la instrumentalización económica y sexual de las mujeres en la posición favorable. Teniendo en cuenta que el $50 \%$ de esta muestra se adhiere al discurso 
regulacionista, frente a un $16 \%$ que se posiciona en contra, podemos preguntarnos ¿qué mecanismos de asimilación consiguen tan exitosamente, que resulte aceptable y convincente la instrumentalización económica y sexual de las mujeres, para posicionarse a favor de la prostitución? Las explicaciones macro nos proporcionan el marco estructural para comprenderlo, pero necesitamos investigaciones micro que nos propongan modelos explicativos de cómo se asimilan cognitivamente argumentos que hacen parecer aceptable, lo que seguramente se rechazaría como valor para otros escenarios.

\section{DOS FORMAS DE LEGITIMAR: HOSTIL Y BENEVOLENTE}

En el estudio que se acaba de referir con una muestra de 239 estudiantes de Psicología, exploramos también la estructura argumentativa subyacente (Delgado y Gutiérrez, 2014). El objetivo es obtener, a partir de las valoraciones otorgadas a las 22 razones extraídas de sus propios discursos, las dimensiones subyacentes que aglutinan los distintos argumentos a partir de las correlaciones entre las puntuaciones que se les otorga. La técnica estadística del Análisis Factorial Exploratorio, nos permite explorar estas dimensiones o factores subyacentes ${ }^{11}$.

Tabla 3.- Solución factorial de argumentos "a favor" (F) y "en contra" (C) en estudiantes

Matriz de componente rotado ${ }^{a}$

\begin{tabular}{|c|c|c|}
\hline & Com & ente \\
\hline & & 2 \\
\hline C10_sexualidad_denigrante & 717 & \\
\hline C1_deshumanizāobjeto & 711 & \\
\hline C7_estigmatiza_mujeres & 655 & \\
\hline C2_sexo_no_mercancía & 648 & \\
\hline C8_siempre_hay_alternativa & 647 & \\
\hline C5_pseudomodernidad & 610 & \\
\hline C9_consentimiento_no_libre & 606 & \\
\hline C11_no_sabe_si_libre & 595 & \\
\hline C4_legalizar_legitima & 594 & \\
\hline C6_riesgos_efectos_negativos & 593 & \\
\hline $\begin{array}{l}\text { 33_explotacion_lucra_otros } \\
\text { F9 no denigrante }\end{array}$ & $15 / 4$ & 773 \\
\hline F4_libērtad_moral_individual & & 740 \\
\hline F1_acuerdo_libre_adultos & & 687 \\
\hline F10_decidir_libremente & & 649 \\
\hline F2_trabajo_cu & & 4 \\
\hline
\end{tabular}

\footnotetext{
${ }^{11}$ Se utilizó el método de Componentes Principales con rotación Varimax. La solución arrojó 3 factores que explican un 52,598\% de la varianza: $21,914 \%$ el primer factor, $15,439 \%$ el segundo factor, y $14,424 \%$ el tercer factor. La fiabilidad, estimada por el coeficiente alfa de Cronbach resultó satisfactoria para los tres factores: 0.88 para el primer factor, 0.78 para el segundo factor, y 0,85 para el tercer factor. La solución factorial resulta por tanto satisfactoria.
} 


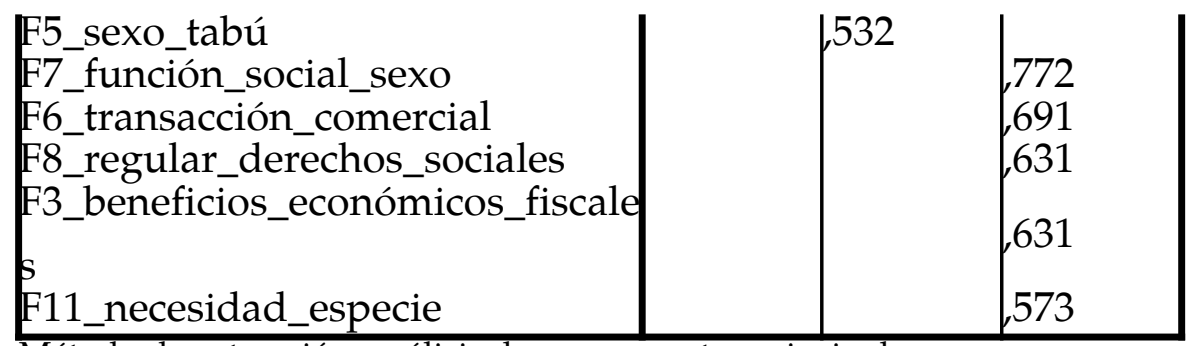

Método de extracción: análisis de componentes principales.

Método de rotación: Varimax con normalización Kaiser. ${ }^{a}$

a. La rotación ha convergido en 6 iteraciones.

Al examinar la estructura emergente, encontramos que los argumentos "en contra" de la prostitución aparecen aglutinados en un factor único (factor 1); es decir, todos ellos se relacionan entre sí aglutinándose en un bloque compacto. Dicho de otro modo, las personas mantienen una tendencia uniforme en las valoraciones que les otorgan, sean éstas altas o bajas. Traduciéndolo al tema que nos ocupa, podemos decir que la posición contraria a la prostitución emerge compactada en el argumentario de esta posición, bien para aceptarla o bien para rechazarla.

En el caso de los argumentos "a favor" emerge una disposición diferente, y esto es lo interesante para el propósito de este análisis. Las razones esgrimidas no aparecen aglutinadas en un único factor, sino que se distribuyen en dos factores independientes como se muestra en la tabla siguiente:

Tabla 4.- Dimensiones subyacentes en los argumentos "A Favor" de la prostitución

\begin{tabular}{|c|c|}
\hline $\begin{array}{l}\text { Factor } 2 \text { (Legitimación blanda } \\
\text { benevolente) }\end{array}$ & Factor 3 (Legitimación dura hostil) \\
\hline F9_No es denigrante para las mujeres & $\begin{array}{l}\text { F7_Cumple una función social: } \\
\text { ofrecer sexo }\end{array}$ \\
\hline $\begin{array}{l}\text { F4_Es cuestión de libertad y moral } \\
\text { individual }\end{array}$ & $\begin{array}{l}\text { F6_Es una transacción comercial } \\
\text { como otras }\end{array}$ \\
\hline F1_Es un acuerdo libre entre adultos & $\begin{array}{l}\text { F8_Regularla garantizaría derechos } \\
\text { sociales }\end{array}$ \\
\hline $\begin{array}{l}\text { F10_Derecho a libre ejercicio y } \\
\text { consumo }\end{array}$ & $\begin{array}{l}\text { F3_Regularla generaría ingresos } \\
\text { fiscales }\end{array}$ \\
\hline F2_Es un trabajo como otro cualquiera & $\begin{array}{l}\text { F11_Es inevitable, necesidad de la } \\
\text { especie }\end{array}$ \\
\hline $\begin{array}{l}\text { F5_ Oponerse es considerar la } \\
\text { sexualidad tabú }\end{array}$ & \\
\hline
\end{tabular}

¿Cómo interpretar este resultado? Examinando la composición de estas 
dimensiones se observa que en una de ellas, factor 3, se aglutinan argumentos que justifican explícitamente la instrumentalización de las mujeres; en ellos, las mujeres prostituidas desaparecen de la escena. El foco está puesto en los actores que obtienen el beneficio de la prostitución, sin que aparezca quién, o a costa de quién, se está obteniendo ese beneficio. La otra dimensión, factor 2, tiene un contenido diferente; aglutina argumentos que explicitan las distintas versiones de una libertad individualista entendida en clave neoliberal; las mujeres entran en la escena, como agentes que participan de la libertad de "profesionalizarse" en la prostitución. Este factor benevolente es coincidente con la categoría "libertad individual", preponderante en el "imaginario profesional" de las posiciones favorables a la prostitución (Barahona, 2015, p.132-133).

La diversificación de la legitimación en los dos factores que se han señalado, se asemeja mucho a la diversificación que Peter Glick y Susan Fiske (1997; 2001) encontraron cuando estudiaron el sexismo. El sexismo, o legitimación de la desigualdad entre mujeres y hombres, puede manifestarse de una forma hostil o de una forma benevolente; pero en ambos casos es una ideología de la desigualdad. Por un lado, el sexismo hostil expresa explícitamente la dominación masculina declarando la superioridad de los hombres y, en el ámbito de las relaciones íntimas, la objetualización de las mujeres como meros instrumentos de placer sexual. El sexismo benevolente, en cambio, se expresa en un formato de magnanimidad, como paternalismo protector, apelando a la complementariedad de los sexos que justifica la diversidad de roles, e idelizando las relaciones íntimas con la exaltación de lo romántico.

El paralelismo entre estas dos dimensiones del sexismo ambivalente, y las dimensiones que emergen en la estructura de la argumentación favorable en nuestro estudio, es una pista muy interesante para comprender cómo se produce la legitimación en el imaginario lego de esta muestra de jóvenes estudiantes de Psicología; estudiantes que, en principio, no tienen ninguna característica especial que los haga diferentes del resto de estudiantes, en lo que a su relación con la prostitución se refiere. En virtud de este paralelismo podemos identificar la primera dimensión como legitimación dura u hostil, por lo que comparte de instrumentalización con el sexismo hostil, y la segunda como legitimación blanda o benevolente por lo que comparte de paternalismo con el sexismo benevolente. Mientras la primera capta la misoginia de imaginarios pre-igualitarios, la segunda pervive en los igualitarios sorteando 
exitosamente las disonancias que produciría el primero. Dicho de otro modo, es una manera más "progre" de mantener la desigualdad. Podemos entonces preguntarnos si la bidimensionalidad de la legitimación de la prostitución, es una forma emergente en la población más joven, a la que se supone más "familiarizada" con el discurso de la igualdad. Dicho de otro modo, ¿se trataría de una forma emergente de representación de la prostitución, que apela a la libertad de elección de las mujeres haciéndola parecer más igualitaria?

Para responder a esta pregunta, realizamos la misma prueba con los datos de la muestra de 2.195 sujetos de 15 a 87 años, 1.417 mujeres (62,5\%) y 849 hombres $(37,5 \%) y$, efectivamente, se obtiene una estructura diferente a la encontrada en la muestra de estudiantes ${ }^{12}$.

Tabla 5.- Solución factorial de argumentos "a favor" (F) y "en contra" (C) en población general

\begin{tabular}{|c|c|}
\hline & Componente \\
\hline & 2 \\
\hline $\begin{array}{l}\text { F1_acuerdo_libre_adultos } \\
\text { F4_libertad_moral } \\
\text { F2_trabajo_cualquiera } \\
\text { F10_decidir_libremente } \\
\text { F11_necesidad_especie } \\
\text { F8_regular_derechos_sociales } \\
\text { F7_función_social_sexo } \\
\text { F6_transacción_comercial } \\
\text { F5_conservadurismo_sexo_tabú } \\
\text { F3_beneficios_económicos } \\
\text { F9_no_denigrante } \\
\text { C10_sexualidad_denigrante } \\
\text { C5_pseudomodernidad } \\
\text { C2__sexo_no_mercancía } \\
\text { C7_estigmatiza_mujeres } \\
\text { C1_deshumaniza_objeto } \\
\text { C8_siempre_hay_alternativa } \\
\text { C4_legalizar_legitima } \\
\text { C9_consentimiento_no_libre } \\
\text { C3_explotación_lucra_otros } \\
\text { C11_no_sabe_si_libre }\end{array}$ & $\begin{array}{l}702 \\
699 \\
684 \\
682 \\
656 \\
648 \\
602 \\
584 \\
577 \\
569 \\
520\end{array}$ \\
\hline
\end{tabular}

\footnotetext{
${ }^{12}$ El AFE realizado por el mismo método de Componentes Principales con rotación Varimax arrojó una solución inicial de 3 factores que explican un 47,100\% de la varianza: 21,431\% el primer factor que aglutina los argumentos "a favor", 19,864\% el segundo factor que aglutina los argumentos "en contra". El tercero, es un factor residual con un 5,214\% de varianza, en el que satura un solo argumento en "contra" referido a los efectos negativos de ejercer la prostitución. Dado que este argumento satura el segundo factor con una carga de .356 se puede considerar que la estructura bifactorial que se muestra en la tabla 5 es la mejor solución. La fiabilidad, estimada por el coeficiente alfa de Cronbach resultó satisfactoria para los dos factores: 0.855 para el primer factor de argumentos "a favor", y 0.846 para el segundo factor de argumentos en contra. La solución factorial resulta por tanto satisfactoria.
} 
\begin{tabular}{l|l|l|l|l|l|}
\hline C6_riesgos_efectos_negativos & J56 \\
\hline Método de extracción: análisis de componentes principales.
\end{tabular}

Método de rotación: Varimax con normalización Kaiser. ${ }^{a}$

a. La rotación ha convergido en 6 iteraciones.

Los argumentos favorables a la prostitución se aglutinan en una única dimensión; no aparece la divergencia entre legitimación hostil y legitimación benevolente. A la luz de estos resultados, pareciera que la legitimación benevolente es un fenómeno emergente, muy sintónico con el discurso regulacionista.

¿Qué podrían estar diciéndonos estos datos? Todo apunta a que es la población joven universitaria, la que mejor ha asimilado una representación benevolente de la prostitución; un imaginario en el que lo relevante es la libertad de elección, la liberacción sexual sin estigmas, y las reglas del mercado; o como la definiera Valerie Jennes (1990), libre del pecado, del crimen y del sexo ilícito. Y como toda representación, la coincidencia con la realidad empírica no es cuestión que afecte. Frente al discurso abolicionista basado en los datos empíricos, el discurso regulacionista basado en las excepciones parece haber seducido con éxito a la joven población universitaria.

\section{CONCLUYENDO}

La mente opera con marcos perceptivos, y éstos -como bien explican las ciencias cognitivas ${ }^{13}$ - filtran los elementos informativos disponibles, para elaborar una representación de la realidad; la percepción es selectiva y está determinada por las categorías con las que se accede a ella. Pero las categorías que configuran la representación las construyen los discursos, no la realidad. El discurso regulacionista ha dotado al imaginario lego de la prostitución, de un marco sintónico -y, por tanto, psicológicamente confortable- con los valores patriarcales y neoliberales que constituyen la atmósfera del sistema. La representación benevolente de este imaginario ingenuo, que parece abrirse paso entre jóvenes estudiantes, resulta muy funcional para dar soporte ideológico a las prácticas de la prostitución en el sistema de valores patriarcal.

Conocemos la realidad siniestra que muestran los datos estadísticos; pero nos

\footnotetext{
${ }^{13}$ Para una aproximación a los marcos perceptivos de la realidad, además de la obra de Daniel Kahneman (2014), las obras de George Lakoff ofrecen una excelente introducción. Entre las traducidas al castellano, puede verse por ejemplo, No pienses en un elefante. Lenguaje y debate político (2007) o Puntos de reflexión. Manual del progresista (2013).
} 
falta encontrar el modo de transformar esta realidad empírica en categorías perceptivas que afecten al imaginario lego sobre la prostitución. La frase atribuida a un funesto personaje de la historia reciente, "una única muerte es una tragedia, pero un millón de muertes es una estadística", captaba con acierto un principio de la psicología social. Lo singular impacta las percepciones sociales de modo diferente a como lo hace lo colectivo, para bien y para mal. ¿Cómo adquiere lo excepcional mayor visibilidad que lo general, en el imaginario social? En la representación mental de la prostitución -así lo sugieren los datos empíricos-, las excepciones cuantitativamente irrelevantes de mujeres que declaran su satisfacción con el "trabajo sexual" empoderante -sin entrar aquí en el análisis de la realidad empírica subyacente en quienes realizan las declaraciones-, tienen mayor saliencia que la abrumadora mayoría de mujeres sexual y económicamente explotadas.

Las investigaciones micro aún tienen mucho que aportar a las construcciones teóricas y a los procesos de formación de imaginarios sociales, para una comprensión más compleja y explicativa de los mecanismos de dominación de las mujeres, en los que se entrecruzan intereses de forma tan eficaz. Hace ya más de 20 años que Sandra Harding hablaba de la necesidad de apropiarse de las intersecciones de las distintas disciplinas para una aproximación científica a la realidad desde el feminismo. La «apropiación de campos entrecruzados» del conocimiento, más que «disolver fronteras de las diferentes disciplinas», es un elemento crucial para poder avanzar, decía en la entrevista realizada por Elizabeth Hirsh y Gary Olson (1995, p.194). Sin duda, esta interdisciplinariedad es un programa de absoluta vigencia para explicar la prostitución y desactivar los mecanismos de reproducción de la desigualdad que tan exitosamente operan en ella.

\section{BIBLIOGRAFÍA}

Baker, Lynda M., Dalla, Rochelle L, y Williansom, Celia (2010). Exiting Prostitution: An Integrated Model. Violence Against Women, 16(5), 579-600. DOI: DOI: $10.1177 / 1077801210367643$

Barahona, María José (2015). Prostitución, Abolicionismo y Trabajo Social. Las actitudes de los y las profesionales de la Comunidad de Madrid. Madrid: 
Universidad Complutense: Tesis doctoral no publicada. Disponible en: http://eprints.ucm.es/36978/

Bauman, Zygmunt (2000). Liquid Modernity. Cambridge: Polity Press. [Traducción: Mirta Rosemberg (2003) Modernidad líquida, México D.F.: Fondo de Cultura Económica]

Butler, Judith (1997). Merely Cultural. Social Text, 52/53 Queer Transexions of Race, Nation, and Gender, 265-277. DOI: 10.1.1.477.2986

Cobo, Rosa (2017). La prostitución en el corazón del capitalismo. Madrid: Catarata.

de Miguel, Ana (2015). Neoliberalismo sexual. El mito de la libre elección. Madrid: Cátedra.

Delgado, Carmen (2014). Viajando a Ítaca por mares cuantitativos. Manual de ruta para investigar en grado y posgrado. Salamanca: Amarú.

Delgado, Carmen, y Gutiérrez, Andrea (2014). Social Representation of Prostitution in Gender Perspective. Procedia- Social and Behavioral Sciences, 161, 82-89. DOI: https://doi.org/10.1016/j.sbspro.2014.12.014

Delgado, Carmen, y Gutiérrez, Andrea (2015). Aproximación cualitativa a las posiciones discursivas sobre la prostitución: perspectiva socio-educativa. Pedagogía Social Revista Interuniversitaria, 26, 35-57. DOI: https://doi.org/10.7179/PSRI_2015.26.02

Delgado, Carmen, y Gutiérrez, Andrea (9-13 septiembre 2014). Violencia simbólica contra las mujeres en el discurso legitimador de la prostitución. IX Congreso Iberoamericano de Psicología. Simposio "La violencia contra las mujeres: experiencias y retos en la prevención y la intervención". Lisboa: Comunicación no publicada.

Farley, Melissa (2003). Prostitution and the Invisibility of Harm. Women y Therapy, 26(3/4), 247-280. DOI: https://doi.org/10.1300/J015v26n03 06

Farley, Melissa (2004). Bad for the body, bad for the heart: Prostitution harms women even if Legalized or Decriminalized. Violence Against Women., 10, 1087-1125. DOI: https:/ / doi.org/10.1177/1077801204268607

Farley, Melissa (2005). Prostitution Harms Women Even if Indoors: Reply to Weitzer. Violence Against Women, 11, 950-964. DOI: https://doi.org/10.1177/1077801205276987

Farley, Melissa (2009). Theory versus reality: Commentary on four articles about trafficking for prostitution. Women's Studies International Forum, 32, 311-315. DOI: doi:10.1016/j.wsif.2009.07.001. 
Farley, Melissa (2017). Risks of Prostitution: When the Person Is the Product. Journal of the Association for Consumer Research, 3(1), Published online December 19, 2017. DOI: http://dx.doi.org/10.1086/695670

Farley, Melissa; Cotton, Ann; Lynne, Jacqueline; Zumbeck, Sybille; Spivak, Frida; Reyes, María E; Álvarez, Dinora y Sezgin, Ufuk (2003). Prostitution and Trafficking in Nine Countries: An Update on Violence and Post-traumatic Stress Disorder. Journal of Trauma Practice Volume, 2(3/4), 33-74. DOI: doi/abs/10.1300/J189v02n03 03

Fraser, Nancy (2013, octubre 14). How feminism became capitalism's handmaiden and how to reclaim it. The Guardian.

Fraser, Nancy (2015). Fortunas del feminismo. Del capitalismo gestionado por el Estado a la crisis neoliberal. Madrid: Traficantes de Sueños.

Gerassi, Lara (2015). A Heated Debate: Theoretical Perspectives of Sexual Exploitation and Sex Work. Journal of Sociology and Social Welfare, 42(4), 79-100. PMCID: PMC4730391

Glick, Peter y Fiske, Susan T. (1997). Hostile and Benevolent Sexism. Psychology of Women Quarterly, 21, 119-135. Doi:10.1111/j.1471-6402.1997.tb00104

Glick, Peter y Fiske, Susan T. (2001). An ambivalent alliance: Hostile and benevolent sexism as complementary justifications for gender inequality. American Psychologist, 56(2), 109-118. Doi:10.1037/0003-066X.56.2.109

Gutiérrez, Andrea (2017). Prostitución: construcción psicosocial de la coacción como consentimiento. Salamanca: Universidad Pontificia de Salamanca. Tesis Doctoral no publicada.

Haraway, Donna (1988). Situated Knowledges: The Science Question in Feminism and the Privilege of Partial Perspective. Feminist Studies, 14(3), 575-599. Doi:10.2307/3178066

Harding, Sandra (1986). The Science Question in Feminism. Londres: Cornell University Press [Traducción Pablo Manzano (1993) Ciencia y Feminismo. Madrid: Morata].

Harding, Sandra (2004). Introduction: Standpoint Theory as a Site of Political, Philosophic, and Scientific Debate. En Sandra Harding, The Feminist Standpoint Theory Reader. Intellectual and Political Controversies (pp. 1-15). New York: Routledge.

Harding, Sandra (2009). Standpoint Theories: Productively Controversial. Hypatia A Journal of Feminist Philosophy, 24(4), 192-200. DOI: 10.1111/j.15272001.2009.01067.x 
Hedin, Ulla-Carin, y Månsson, Svel-Axen (2003). The Importance of Supportive Relationships among Women Leaving Prostitution. En Melissa Farley, Prostitution, Trafficking and Traumatic Stress (pp. 223-238). New York: The Haworth Press.

Heider, Fritz (1958). The psychology of interpersonal relations. New York: Wiley.

Hirsh, Elizabeth y Olson, Gary (1995). Starting from Marginalized Lives: A Conversation with Sandra Harding. Journal of Composition Theory, 15(2), 193225. Disponible online

Jeffreys, Sheila (2012). Beyond 'Agency' and 'Choice' in Theorizing Prostitution. En Maddy Coy (Ed.), Prostitution, Harm and Gender Inequality (pp. 58-69). Londres: Ashgate Publishing.

Jenness, Valerie (1990). From Sex as Sin to Sex as Work: COYOTE and the Reorganization of Prostitution as a Social Problem. Social Problems, 37(3), 403420. DOI:10.2307/800751

Gimeno, Beatriz (2012). La prostitución. Barcelona: Bellaterra.

Kahneman, Daniel (2011). Thinking, Fast and Slow. Londres: Penguin Books [Traducción: Joaquín Chamorro (2014). Pensar rápido, pensar despacio. Barcelona: Debate]. Disponible versión online.

Kelly, George A. (1955). The psychology of personal constructs. New York: Norton.

Kraus, Ingeborg (2016, Octubre, 5). "Trauma and Prostitution: Le traumatisme comme pré-condition et conséquence de la prostitution". Montreal (Canadá). [Traducción: Olga Baselga "El trauma como requisito previo y como consecuencia de la prostitución" Traductoras por la abolición de la prostitución]

Lakoff, George (2007). No pienses en un elefante. Lenguaje y debate político. Madrid: Complutense.

Lakoff, George (2013). Puntos de reflexión. Manual del progresista. Barcelona: Península.

Leigh, Carol (1997). Inventing sex work. En Jill Nagel, Whores and Other Feminists (pp. 226-231). New York: Routledge.

León, Ofelio G., y Montero, Ignacio (2010). Métodos de Investigación en Psicología y Educación. Madrid: McGraw-Hill.

Lorente, Miguel (22 de mayo de 2013). El postmachismo I. El País, Obtenido de: $\underline{\text { htp:// }}$ blogs.elpais.com/autopsia/2013/05/el-posmachismo-i.html

Månsson, Svel-Axen, y Hedin, Ulla-Carin (1999). Breaking the Matthew Effect on Women Leaving Prostitution. International Journal of Social Welfare, 8(1), 67-77. Doi:10.1111/1468-2397.00063 
Martín Baró, Ignacio (1990). Acción e Ideología. Psicología Social desde Centroamérica. (4 $4^{\mathrm{a}}$ Ed.). San Salvador (El Salvador): UCA Editorial.

Mertuil, Morgane (2017). Liberad el feminismo. Barcelona: Bellaterra.

Ministerie van Justitie in Veiligheid (2015). Prostitution in the Netherlands 2014 (Vols.

Cahiers 2015-01). La Haya: WODC. Disponible version online

Nagel, Jill (1997). Whores and Other Feminists. New York: Routledge.

Niemi, Johanna (2010). What we talk about when we talk about buying sex. Violence Against Women, 16(2), 159-172. Doi: 10.1177/1077801209355239

Niemi, Jiemi, y Aaltonen, Jussi (2017). Tackling Trafficking by Targeting Sex Buyers: Can It Work? Violence Against Women, 23(10), 1228-1248. Doi:10.1177/1077801216657896

Pedernera, Laura, y Torrado, Esther (2015). La Prostitución desde la Perspectiva de la Demanda: Amarres Enunciativos para su Conceptualización. Oñati Socio-legal Series [online], 5(5), 1382-1400. Disponible online

Ramírez, Laura, y Levy, Shery (2010). Sentido común y conflicto: impacto de las teorías legas sobre relaciones intergrupales. Universitas Psychologica, 9(2), 331-343. Disponible online

Raphael, Jody, y Shapiro, Deborah L. (2004). Violence in indoor and outdoor prostitution venues. Violence Against Women, 10, 126-139. Doi:10.1177/1077801203260529

Raymond, Janice (2004). Prostitution on demand: Legalizing the buyers as sexual consumers. Violence Against Women, 10, 1156-1186.

Schulze, Erika, Novo, Sandra I., Mäson, Peter, y Saklin, Maria (2014). Sexual exploitation and prostitution and its impact on gender equality. Bruselas: European Parliament. Disponible en: http://www.europarl.europa.eu/studies

Segato, Rita L. (2010). Las estructuras elementales de la violencia. Buenos Aires: Prometeo.

Silbert, Mimi H., y Pines, Ayala M. (1982). Victimization of Street Prostitutes. Victimology, 7(1-4), 122-133.

TowandaRebels. (2017). "Hola, Putero". https://youtu.be/cb7t10c-bIM

Vanwesenbeeck, Ine (1994). Prostitutes' well-being and risk. Amsterdam: VU University Press.

Weitzer, Ronald (1994). Community groups and prostitutes. The Gauntlet, 1(7), 121124.

Weitzer, Ronald (2005). Flawed Theory and Method in Studies of Prostitution. Violence Against Women, 11(7), 934-949. DOI: 10.1177/1077801205276986 
Yanes, Zenaida (2012). Cómo ver el mundo desde el feminismo. Una reflexión sobre la objetividad científica. Memorias IX Congreso Iberoamericano de Ciencia, Tecnología y Género. Sevilla: OEI-AECID. Disponible online 